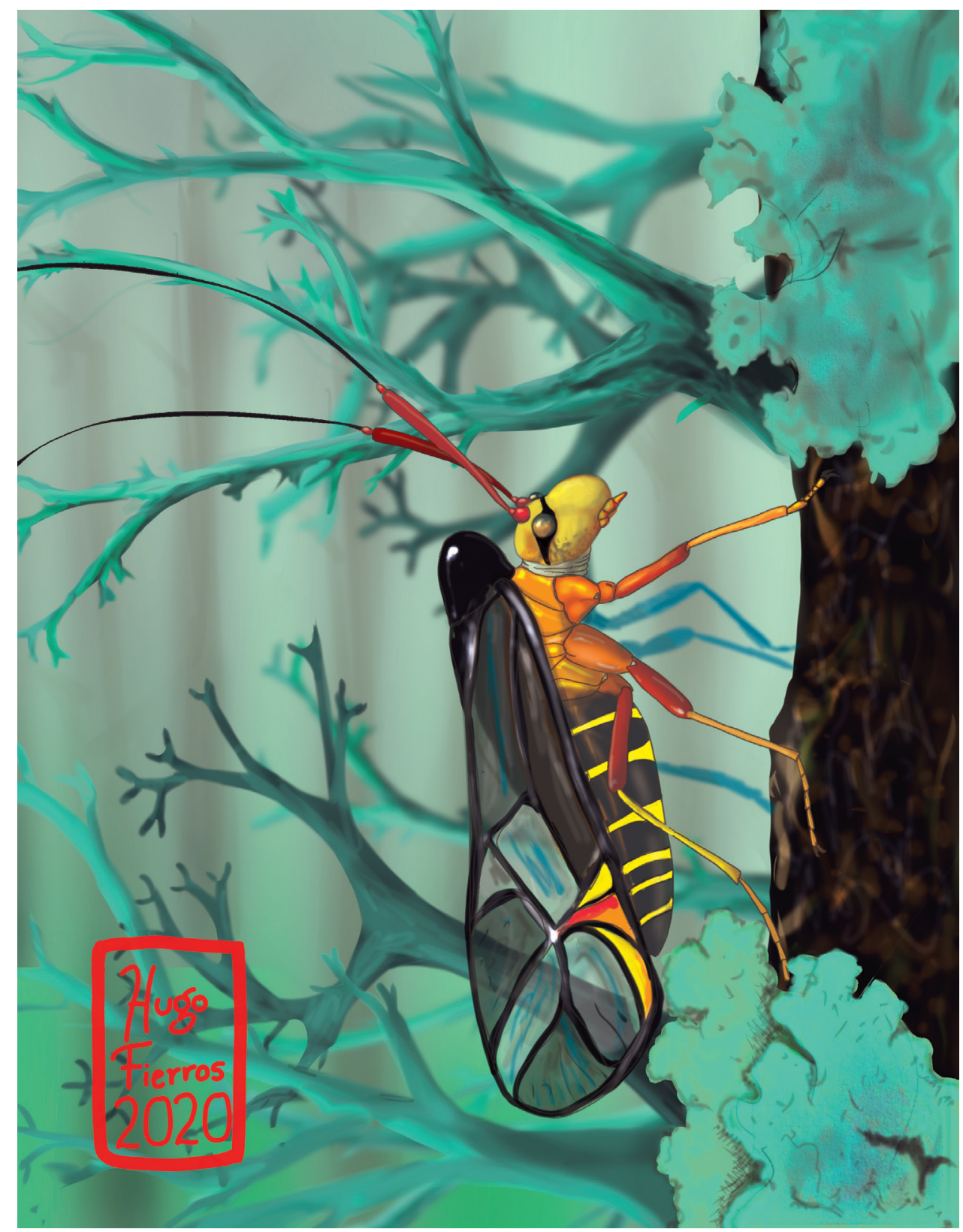

Dugesiana, Año 27, No. 2, julio 2020-diciembre 2020 segundo semestre de 2020), es una publicación semestral, editada por la Universidad de Guadalajara, a través del Centro de Estudios en Zoología, por el Centro Universitario de Ciencias Biológicas y Agropecuarias. Camino Ramón Padilla Sánchez \# 2100, Nextipac, Zapopan, Jalisco, Tel. 37771150 ext. 33218, http://148.202.248.171/dugesiana/index.php/DUG/index, glenusmx@gmail.com. Editor responsable: José Luis Navarrete-Heredia. Reserva de Derechos al Uso Exclusivo 04-2009-062310115100203, ISSN: 2007-9133, otorgados por el Instituto Nacional del Derecho de Autor. Responsable de la última actualización de este número: José Luis Navarrete-Heredia, Editor y Ana Laura González-Hernández, Asistente Editorial. Fecha de la última modificación 1 de julio 2020, con un tiraje de un ejemplar.

Las opiniones expresadas por los autores no necesariamente reflejan la postura del editor de la publicación.

Queda estrictamente prohibida la reproducción total o parcial de los contenidos e imágenes de la publicación sin previa autorización de la Universidad de Guadalajara. 
Nota científica

\section{Trigonopeltastes frontalis Bates, 1889 y Plesiorutela specularis (Bates, 1888) (Coleoptera: Scara- baeoidea: Scarabaeidae) en Campeche, México}

\section{Trigonopeltastes frontalis Bates, 1889 and Plesiorutela specularis (Bates, 1888) (Coleoptera: Scarabaeoi- dea: Scarabaeidae) in Campeche, Mexico}

\section{Eduardo Rafael Chamé-Vázquez ${ }^{1 *}$ y Noemí Salas-Suárez ${ }^{2}$}

${ }^{1}$ Colección de Insectos. El Colegio de la Frontera Sur (Unidad Tapachula). Carretera Antiguo Aeropuerto Km. 2,5, C.P. 30700, Tapachula, Chiapas, México. echame@ecosur.mx, e_chame@yahoo.com; ${ }^{2}$ Museo de Zoología. El Colegio de la Frontera Sur (Unidad Chetumal). Av. Centenario Km. 5,5, C.P. 77014, Chetumal, Quintana Roo, México,nsalas@ecosur.mx,*Autor de correspondencia

En México, la fauna de escarabajos fitófagos (Scarabaeidae: "Pleurosticti") se encuentra compuesta por 1,179 especies, distribuidas en 127 géneros de las subfamilias Melolonthinae, Rutelinae, Dynastinae, Cetoniinae y Trichiinae; así mismo, destacan los estados de Chiapas, Durango, Guerrero, Hidalgo, Jalisco, México, Oaxaca, Puebla y Veracruz por ser las entidades federativas con mayor riqueza de especies (Morón et al. 2014). Históricamente, Campeche ha sido considerado un estado con baja representatividad taxonómica en este grupo de escarabajos (Morón et al. 1997, 2014), puesto que son escasos los estudios que se han realizado con estos coleópteros. No obstante, el trabajo de Morón-Ríos y Morón (2016) dejó en claro que la riqueza de escarabajos fitófagos en este estado es mayor a lo que se conoce actualmente. Los muestreos sistemáticos que estos autores realizaron en la Reserva de Calakmul dieron a conocer un total de 29 especies de Scarabaeidae "Pleurosticti", siendo el 45\% de ellos nuevos registros para el estado de Campeche. Teniendo en cuenta el conocimiento que se tiene de esta familia de escarabajos en los estados de Quintana Roo y Yucatán, no es de sorprenderse que se sigan adicionando nuevas especies al listado de Campeche. Por tal motivo, el objetivo de este trabajo es informar sobre la presencia de dos nuevos registros y aportar un listado de las especies de escarabajos fitófagos que actualmente están registrados para el estado de Campeche.

Los ejemplares estudiados se encuentran depositados en la Colección de Insectos de El Colegio de la Frontera Sur en Tapachula, Chiapas (registro SEMARNAT CHISINV-133-09-02, acrónimo ECO-TAP-E).

\section{Trigonopeltastes frontalis Bates, 1889} Trichiinae: Trichiini

Esta especie se reconoce por el clípeo enteramente negro, un parche de setas cortas semierectas de color ocre en la base del clípeo, triángulo pronotal completo, pruinoso, de color amarillo cremoso, protibia con dos dientes prominentes en su borde exterior, protibia y mesotibia de color ocre, los élitros con una coloración ocre o anaranjado extendiéndose más allá de dos marcas transversales cretáceas y el pigidio con marcas cretáceas no interrumpidas en la base y extendidas hacia los lados (Smith 2016). Su distribución comprende México, Belice, Guatemala, Honduras y El Salvador (Fig. 1). Se ha recolectado en una variedad de hábitats conservados o en comunidades secundarias establecidas entre los 100 y $1500 \mathrm{~m}$ de altitud. Los adultos vuelan entre mayo y agosto, los cuales se alimentan de diversas flores, como Casearia aculeata Jacq. (Salicaceae) y Solidago sp (Compositae). En México se ha registrado de Chiapas, Veracruz, San Luis Potosí, Tamaulipas y Nuevo León (Cave 1983, Howden 1968, Morón et al. 1997, Smith 2016). En este trabajo se registra por primera vez para el estado de Campeche.

Nuevos registros: [Museo de Zoología ECO-CH-C] México: Campeche. Mpio. Calakmul. Camino a la zona arqueológica de Nadzcan. 23/V/2002. J. L. Cozar. C. 0937 (1 +). México: Campeche. Mpio. Calakmul. Ejido Nuevo Becal, camino a Flores Magón. 3/V/2002. J. L. Cozar. C. 0991 (1 今̈; Fig. 2).

\section{Plesiorutela specularis (Bates, 1888) Rutelinae: Rutelini}

Esta especie se distingue por tener una coloración dorsal negra brillante con franjas rojo escarlata en cabeza, pronoto, élitros y pigidio, ápice del clípeo con dos proyecciones agudas, placa unguitractora expuesta más allá de la base de las uñas y carente de setas, élitros ausentes de estrías y presencia de una fóvea lateral en el pronoto (Jameson 1997). Habita en el bosque tropical perennifolio y selva mediana subperenifolia establecida entre el nivel del mar y los $200 \mathrm{~m}$ de altitud. A los adultos se les ha colectado en flores de Guazuma ulmifolia Lam. (Sterculiaceae) y están activos durante el día, entre abril y julio. Es una especie rara con distribución en México, Belice, Honduras y Guatemala (Fig. 1). En México se ha registrado en Veracruz, Chiapas, Tabasco, Quintana Roo y Yucatán (Jameson 1997, Le Tirant 
2003, Reyes-Novelo y Morón 2005, Sánchez, 2017). Se registra por primera vez para el estado de Campeche.

Nuevo registro: México: Campeche. Mpio. Calakmul. Zona J. Calakmul. 19/VII/1999. S. Uc. C. 0267 (19; Fig. $3)$.

Con la adición de estos nuevos registros, la fauna de Scarabaeidae Pleurosticti en Campeche se compone de 43 especies repartidas en 23 géneros y cinco subfamilias (Cuadro 1).

\section{LITERATURA CITADA}

Cave, R.D. 1983. New records of Trigonopeltastes in El Salvador (Coleoptera: Scarabaeidae). The Coleopterist Bulletin, (37): 152.

Chamé-Vázquez, E.R. y N. Salas-Suárez. 2018. Nuevos registros estatales de Euphoria Burmeister en México. Revista Chilena de Entomología, 44(4):467-469.

Howden, H.F. 1968. A review of the Trichiinae of North and Central America (Coleoptera: Scarabaeidae). Memoirs of the Entomological Society of Canada, (54): 1-77.

Jameson, M.L. 1997. Phylogenetic analysis of the subtribe Rutelina and revision of the Rutela generic groups (Coleoptera: Scarabaeidae: Rutelinae: Rutelini). Bulletin of the University of Nebraska State Museum, (14): 1-183.

Le Tirant, S. 2003. A new country record for Plesiorutela specularis (H. Bates) (Scarabaeidae: Rutelinae). Dugesiana, 10(1): 30 .

Moore, M.R., M.L. Jameson, B.H. Garner, C. Audibert, A.B.T. Smith and M. Seidel. 2017. Synopsis of the pelidnotine scarabs (Coleoptera, Scarabaeidae, Rutelinae, Rutelini) and annotated catalog of the species and subspecies. ZooKeys, (666): 1-349.

Moore, M.R., R.D. Cave and M.A. Branham. 2018. Annotated catalog and bibliography of the cyclocephaline scarab beetles (Coleoptera, Scarabaeidae, Dynastinae, Cyclocephalini). Zookeys, (745): 101-378.

Morón, M. A.1999. Four new species of Phyllophaga Harris (Coleoptera: Melolonthidae, Melolonthinae) from Southeastern Mexico. The Coleopterist Bulletin, 53(4): 377-387.

Morón, M.A. 2018. Revisión del grupo de especies "pruinosa" de Phyllophaga (Phytalus) Coleoptera: Melolonthidae: Melolonthinae). Dugesiana, 25 (1): 37-59.

Morón-Ríos, A. y M.A. Morón. 2016. Evaluación de la fauna de Coleoptera Scarabaeoidea en la Reserva de la Biósfera de Calakmul, Campeche, México. Southwestern Entomologist, 41(2):469-484.

Morón, M. A., Ratcliffe, B. C. y C. Deloya (Eds). 1997. Atlas de los escarabajos de México (Coleoptera: Lamellicornia), Vol. 1. Familia Melolonthidae. CONABIO-SM,

Recibido: 10 de octubre 2019

Aceptado: 6 de febrero 2020
México, D. F. 278 pp.

Morón, M. A., G. Nogueira, C.V. Rojas-Gómez y R. Arce-Pérez. 2014. Biodiversidad de Melolonthidae (Coleoptera) en México. Revista Mexicana de Biodiversidad, Supl. 85: 298-302.

Orozco, J. 2012. Monographic revision of the american genus Euphoria Burmeister, 1842 (Coleoptera: Scarabaeidae: Cetoniinae). The Coleopterists Bulletin, 66(mo4): 1-182.

Ratcliffe, B.C. 2013. A revision of the neotropical genus Amithao Thomson, 1878 (Coleoptera: Scarabaeidae: Cetoniinae: Gymnetini). The Coleopterists Bulletin, 67(3): 265-292.

Reyes-Novelo, E. y Morón, M.A. 2005. Fauna de Coleoptera Melolonthidae y Passalidae de Tzucacab y Conkal, Yucatán, México. Acta Zoológica Mexicana (n.s.), 21(2): 15-49.

Sánchez S., S. 2017. Plesiorutela specularis (Bates, 1888) (Coleoptera: Scarabaeidae): primer reporte para Tabasco, México. Revista Nicaragüense de Entomología, (126): $1-8$.

Smith, A.B.T. 2016. Five new species of Trigonopeltastes Burmeister and Schaum from Central America with new country records for other New World Trichiini (Coleoptera: Scarabaeidae: Cetoniinae). ZooKeys, (617): 91-127.

Vaurie, P. 1960. A revision of the genus Diplotaxis (Coleoptera, Scarabaeidae, Melolonthinae), Part II. Bulletin of the American Museum of Natural History, 120(2): 161-434.

Cuadro 1. Listado de especies de escarabajos fitófagos presentes en el estado de Campeche, México. Los nuevos registros se indican con un asterisco $(*)$. Se indica la distribución conocida en México: Ags=Aguascalientes, BCS $=$ Baja California Sur, Camp $=$ Campeche, $\mathbf{C h i s}=$ Chiapas, $\mathbf{C o a}=$ Coahuila, $\mathbf{C o l}=$ Colima, $\mathbf{D F}=$ Distrito Federal, Gua $=$ Guanajuato, Gro $=$ Guerrero, Hgo $=$ Hidalgo, Jal=Jalisco, Mex=Estado de México, Mich=Michoacán, Mor=Morelos, Nay=Nayarit, $\mathbf{N L}=$ Nuevo León, Oax $=$ Oaxaca, Pue $=$ Puebla, Quer=Querétaro, $\mathbf{Q R}=$ Quintana Roo, Sin $=-$ Sinaloa, Son $=$ Sonora, SLP $=$ San Luis Potosi, Tab $=$ Tabasco, Tamps $=$ Tamaulipas, Ver $=$ Veracruz, Yuc $=$ Yucatán, Za$\mathbf{c}=$ Zacatecas. Referencias: ${ }^{1}$ Vaurie (1960), ${ }^{2}$ Morón et al. (1997), ${ }^{3}$ Morón (1999), ${ }^{4}$ Orozco (2012), ${ }^{5}$ Ratcliffe (2013), ${ }^{6}$ Morón-Ríos y Morón (2016), ${ }^{7}$ Moore et al. (2017), ${ }^{8}$ Chamé-Vázquez y Salas-Suárez (2018), ${ }^{9}$ Morón (2018), ${ }^{10}$ Moore et al. (2018).

\section{SCARABAEIDAE: "PLEUROSTICTI" Melolonthinae}

Diplotaxis denigrata Bates, $1889^{1}$ Camp, Chis, Mich, Oax, Pue, QR, Ver, Yuc Phyllophaga (Chlaenobia) vexata Horn, $1885^{2,6}$ Camp, Hgo, Pue, QR, SLP, Tab, Ver, Yuc 

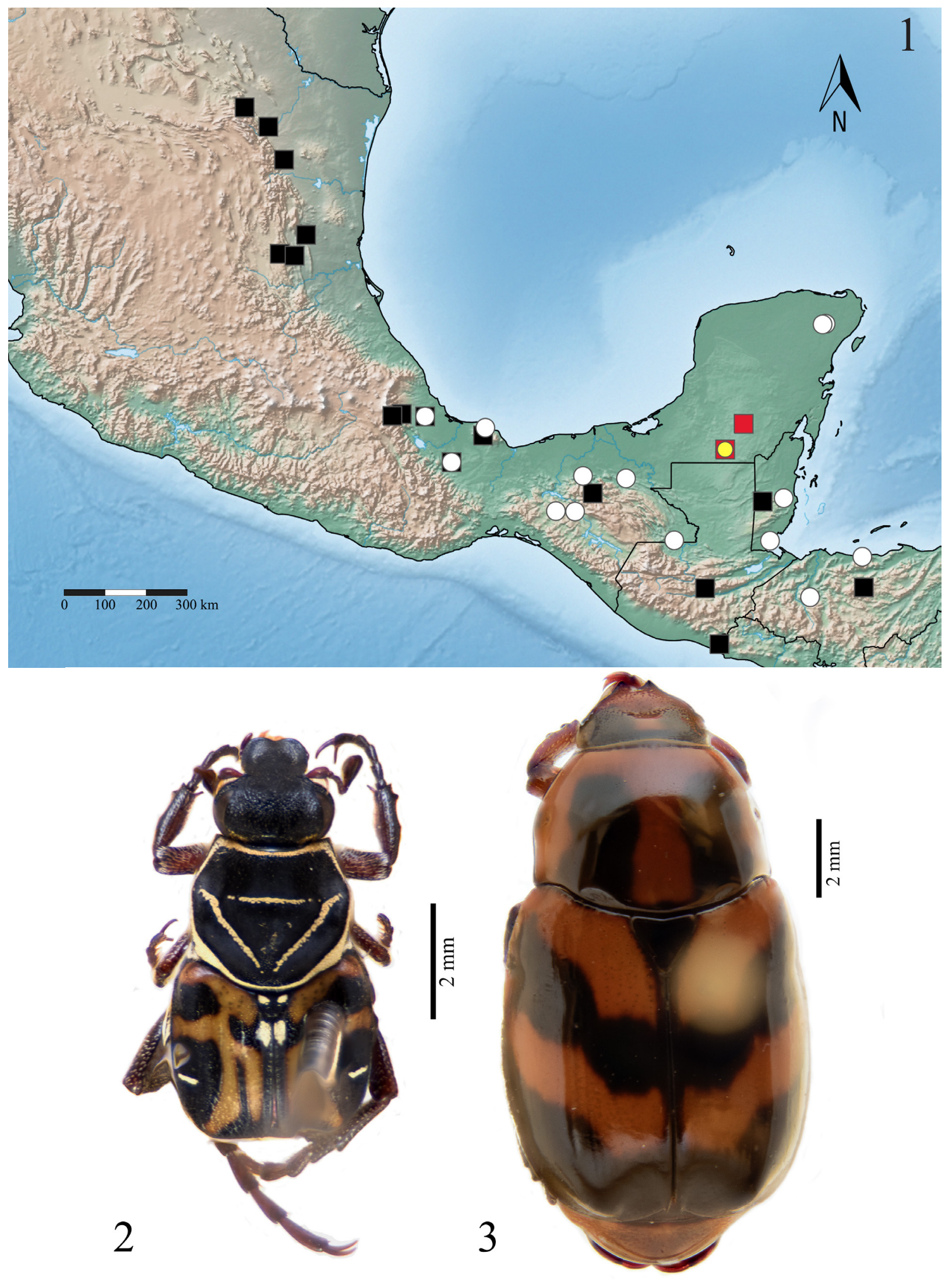

Figura 1. Distribución conocida de T. frontralis (cuadros negros) y P. specularis (círculos blancos. Los nuevos registros se indican en color rojo (T. frontalis) y amarillo (P. specularis). Figura 2. Vista dorsal de Trigonopeltastes frontalis Bates (macho). Figura 3. Vista dorsal de Plesiorutela specularis (Bates) (hembra). 
Phyllophaga (Phytalus) pruinosa (Blanchard, 1851) ${ }^{9}$

Camp, Chis, Hgo, Oax, Pue, SLP, Tamps, Ver

Phyllophaga (Phyllophaga) caanchaki Morón, $1998^{6}$

Camp, Chis, QR, Yuc

Phyllophaga (Phyllophaga) menetriesi (Blanchard, 1851) ${ }^{6}$

Camp, Chis, Oax, Pue, Ver

Phyllophaga (Phyllophaga) pallidicornis (Moser, 1921) 2,6

Camp, QR, Yuc

Phyllophaga (Phyllophaga) pseudocarus Morón, $1999^{3}$

Camp, Yuc

Phyllophaga (Phyllophaga) sturmi (Bates, 1888) 2,6

Camp, Chis, Oax, Pue, Tab, Ver

Phyllophaga (Phyllophaga) testaceipennis (Blanchard, 1850) ${ }^{2}$

Camp, Chis, Hgo, Oax, Pue, Tab, Ver

\section{Rutelinae}

Chasmodia collaris (Blanchard, 1835) ${ }^{6}$

Camp, Chis, Ver, Yuc

Macropoides c. crassipes (Horn, 1866) ${ }^{6}$

Camp, Chis, Oax, Ver, Yuc

Pelidnota centroamericana Ohaus, $1913^{6,7}$

Camp, Chis, QR, Tab, Ver, Yuc

Pelidnota punctulata Bates, $1888^{6,7}$

Camp, Chis, Oax, Pue, QR, Tab, Ver, Yuc

Pelidnota strigosa Laporte, $1840^{7}$ Ver

Camp, Chis. Coa, DF, Hgo, Oax, Pue, SLP, Tab, Tamps,

Plesiorutela specularis (Bates, 1888) *

Chis, QR, Tab, Ver, Yuc

\section{Dynastinae}

Cyclocephala gravis Bates, $1888^{6,10}$

Camp, Chis, Gua, Oax, QR, SLP, Tab, Ver, Yuc

Cyclocephala lunulata Burmeister, $1847^{6,10}$

Ags, BCS, Camp, Chis, Coa, Col, DF, Gua, Gro, Hgo,

Jal, Mex, Mich, Mor, Nay, NL, Oax, Pue, Quer, QR,

Sin, Son, SLP, Tab, Tamps, Ver, Yuc, Zac

Cyclocephala sparsa Arrow, $1902^{6,10}$

Camp, Chis, Gro, Hgo, Jal, Mex, Mich, Mor, Nay, Oax,

QR, Ver, Yuc

Coelosis biloba (Linnaeus, 1767) ${ }^{6}$

Camp, Chis, Oax, QR, Ver, Yuc

Dyscinetus dubius (Olivier, 1789) ${ }^{10}$

Camp, Chis, DF, Gro, Jal, Nay, Oax, QR, SLP, Sin, Tab,

Tamps, Tlax, Ver

Dyscinetus laevipunctatus Bates, $1888^{10}$

Camp, Chis, Coa, Col, DF, Gro, Jal, Mich, Nay, NL,

Oax, Pue, QR, SLP, Sin, Tab, Tamps, Ver, Yuc

Enema endymion Chevrolat, $1843^{6}$

Chis, Hgo, Oax, Tab, Tamps, Ver, Yuc
Homophileurus quadrituberculatus (Beauvois, 1806) 2,6

Camp, Chis, Tab

Megasoma elephas (Fabricius, 1775) ${ }^{2,6}$

Camp, Chis, Col, Gro, Jal, Mich, Nay, Oax, Sin, Tab,

Ver, QR, Yuc

Phileurus didymus (Linnaeus, 1758) ${ }^{2}$

Camp, Chis, Jal, Gro, Hgo, Mich, Nay, Oax, Pue, QR,

SLP, Tab, Ver, Yuc

Stenocrates canuli Delgado, $1991^{6,10}$

Camp, Chis, Oax, QR, Ver, Yuc

Stenocrates bicarinatus Robinson, $1948^{6,10}$

Camp, Chis, Gro, Jal, Oax, Pue, SLP, Tab, Ver

Strategus aloeus (Linnaeus, 1758) ${ }^{6}$

Ags, BCS, Camp, Chis, Coa, Col, DF, Gua, Gro, Hgo, Jal, Mex, Mich, Mor, Nay, NL, Oax, Pue, Quer, QR, Sin, Son, SLP, Tab, Tamps, Ver, Yuc, Zac

Tomarus nasutus (Burmeister, 1847) ${ }^{2}$

Camp, Chis, Gro, Jal, Mich, Oax, QR, Tamps, Yuc

\section{Cetoniinae}

Cotinis viridicyanea $\left(\right.$ Perbosc, 1839) ${ }^{2}$

Camp, Yuc

Cotinis subviolacea (Gory \& Percheron, 1833) ${ }^{6}$

Camp, Chis, Oax, Ver

Amithao cavifrons (Burmeister, 1842) ${ }^{5,6}$

Camp, Chis, Hgo, Oax, Ver, Yuc

Amithao erythropus (Burmeister, 1842) 5,6

Camp, Chis, Tab, Ver, Yuc

Balsameda pulverulenta (Burmeister, 1842) ${ }^{6}$

Camp, Chis, Gro, QR, Ver, Yuc

Euphoria leprosa Burmeister, $1842^{8}$

Camp, QR, Tab, Ver, Yuc

Euphoria pulchella (Gory \& Percheron, 1833) ${ }^{4}$

Camp, Chis, Col, DF, Mex, Gro, Jal, Mich, Mor, Nay,

Oax, Pue, Sin, Son, Ver, Yuc, Zac

Gymnetis caseyi Antoine, $2001^{6}$

Camp

Gymnetis chevrolat sallei Schaum, $1844^{6}$

Camp, Hgo, Oax, Pue, QR, Ver, Yuc

Gymnetis difficilis Burmeister, $1842^{6}$

Camp, Chis, Oax, Ver, Yuc

Hoplopyga liturata (Olivier, 1789) ${ }^{6}$

Camp, Chis, Hgo, Jal, Oax, Pue, QR, Ver, Yuc

Hoplopyga ocellata (Gory \& Percheron, 1833) ${ }^{6}$

Camp, QR, Tab, Yuc

Trichiinae

Trigonopeltastes frontalis Bates, 1889 *

Chis, NL, SLP, Tamps, Ver

Trigonopeltaste geometrica Schaum, $1841^{2}$

Camp, Chis, SLP, Tab, Ver 